Determination of laser damage initiation probability and growth on fused silica scratches

M. A. Norton, C. W. Carr, D. A. Cross, R. A. Negres, J. D. Bude, W. A. Steele, M. V. Monticelli, T. I. Suratwala

November 15, 2010

SPIE Laser Damage Conference

Boulder, CO, United States

September 26, 2010 through September 29, 2010 
This document was prepared as an account of work sponsored by an agency of the United States government. Neither the United States government nor Lawrence Livermore National Security, LLC, nor any of their employees makes any warranty, expressed or implied, or assumes any legal liability or responsibility for the accuracy, completeness, or usefulness of any information, apparatus, product, or process disclosed, or represents that its use would not infringe privately owned rights. Reference herein to any specific commercial product, process, or service by trade name, trademark, manufacturer, or otherwise does not necessarily constitute or imply its endorsement, recommendation, or favoring by the United States government or Lawrence Livermore National Security, LLC. The views and opinions of authors expressed herein do not necessarily state or reflect those of the United States government or Lawrence Livermore National Security, LLC, and shall not be used for advertising or product endorsement purposes. 


\title{
Determination of laser damage initiation probability and growth on fused silica scratches
}

\author{
Mary A. Norton*, C. Wren Carr, David A. Cross, Raluca A. Negres, Jeffrey D. Bude, William A. \\ Steele, Marcus V. Monticelli, Tayyab I. Suratwala \\ Lawrence Livermore National Laboratory, 7000 East Ave., Livermore, CA, USA 94551-9234 \\ *corresponding author: mnorton@1lnl.gov
}

\begin{abstract}
Current methods for the manufacture of optical components inevitably leaves a variety of sub-surface imperfections including scratches of varying lengths and widths on even the finest finishes. It has recently been determined that these finishing imperfections are responsible for the majority of laser-induced damage for fluences typically used in ICF class lasers. We have developed methods of engineering subscale parts with a distribution of scratches mimicking those found on full scale fused silica parts. This much higher density of scratches provides a platform to measure low damage initiation probabilities sufficient to describe damage on large scale optics. In this work, damage probability per unit scratch length was characterized as a function of initial scratch width and post fabrication processing including acidbased etch mitigation processes. The susceptibility of damage initiation density along scratches was found to be strongly affected by the post etching material removal and initial scratch width. We have developed an automated processing procedure to document the damage initiations per width and per length of theses scratches. We show here how these tools can be employed to provide predictions of the performance of full size optics in laser systems operating at $351 \mathrm{~nm}$. In addition we use these tools to measure the growth rate of a damage site initiated along a scratch and compare this to the growth measured on an isolated damage site.
\end{abstract}

Keywords: Surface damage, laser damage growth, laser damage fused silica, fused silica scratches

\section{INTRODUCTION}

The reduction of laser damage initiation to achieve long working lifetimes for fused silica $\left(\mathrm{SiO}_{2}\right)$ optics used in UV lasers with nanosecond pulses has been the subject of investigation for many years. Improvements in the fabrication process over the decades from 1997 to 2007 resulted in two orders of magnitude reduction in damage initiations at 351 $\mathrm{nm}$ for working fluences up to $12 \mathrm{~J} / \mathrm{cm}^{2}$ and nanosecond pulse widths [1]. With this work came the realization that even with greatly improved finishing techniques there still remains a distribution of scratches on the surfaces. More recent work on further reducing the impact of residual scratches on the damage initiation has utilized chemical etch techniques and again two orders of magnitude improvement has been achieved. The details of this Advanced Mitigation Process (AMP) is described in more detail elsewhere [2], [3].

With the current AMP treatment, and for fluences up to about $12 \mathrm{~J} / \mathrm{cm}^{2}$, the damage initiations along a length of scratch are expected to be only on the order of 1 per meter for scratches having a width of about $30 \mu \mathrm{m}$. Thus to evaluate the effectiveness of the AMP treatment with off-line testing of surfaces it is necessary to test samples with scratch distributions providing sufficient lengths to achieve statistically significant sampling. To achieve this we have developed new techniques to provide and test subscale parts with scratches having the same damage response as a function of laser conditions, but at a much higher density, than that found on production parts. 


\section{EXPERIMENTAL}

\subsection{Experiment overview}

Since damage initiations at working fluences will be dominated by the scratch density on the fabricated optic we have developed methods to prepare samples with realistic scratches. The samples once fabricated must be tested under the desired laser conditions and then results must be quantified. This requires methodologies to measure scratch lengths and widths and to identify damage induced by the laser shots.

\subsection{Sample preparation}

For off-line evaluation of various surface preparations it is convenient to utilize 2-inch fused silica substrates. The 1-cm thick samples were scratched by sliding a sample over a dry polyurethane pad loaded with a number of fused silica $200 \mu \mathrm{m}$ spheres; this is illustrated in Figure 1a. The resulting scratches are easily revealed after a light etch as shown in the micrograph of Figure 1b. It should be noted that even the best conventionally finished optic has scratches which are only revealed by a light etch. [2]

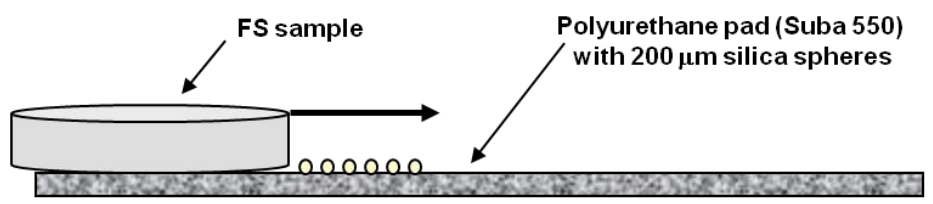

(a)

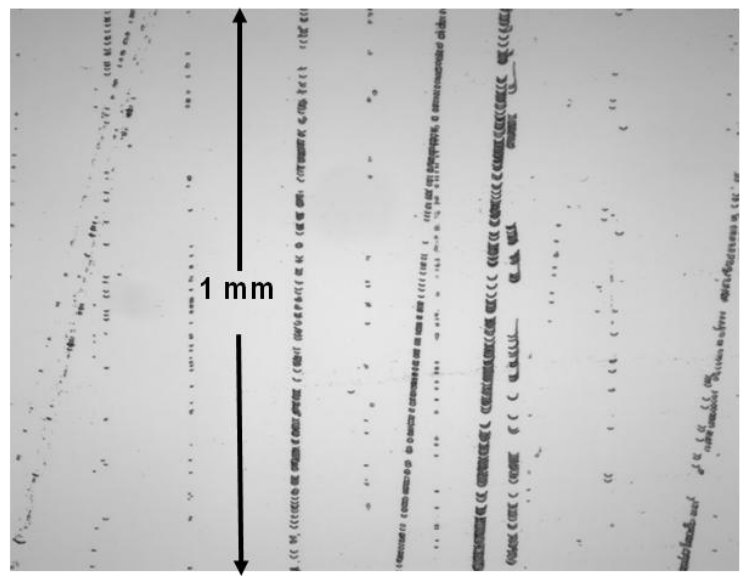

(b)

Figure 1. Scratch sample technique and micrograph.

Each sample prepared with this technique has a unique array of scratches but they have in common a distribution of lengths and widths that encompass those scratches that might be present on a full size optic. The scratch morphology is primarily composed of brittle, trailing Hertzian fractures. The method produces mostly parallel lines of scratches which ease the measurement of lengths and widths of the ensemble. A typical part will have a total scratch length of over a meter in the region that is ultimately tested with the laser as will be described below.

\subsection{Laser testing}

The laser damage testing procedure is an adaptation to our previously developed technique for studying isolated damage sites [4]. In order to test a statistically representative scratch distribution we use the Optical Sciences Laser (OSL) [5] which provides a $3-\mathrm{cm}$ beam on target at fluence and pulse shapes of interest. The OSL is a master oscillator power amplifier (MOPA) system producing $200 \mathrm{~J}$ of light at $1.053 \mu \mathrm{m}$ which is frequency converted to $\sim 100 \mathrm{~J}$ at $351 \mathrm{~nm}$. Pulse 
width is adjustable from $\sim 100$ ps to $\sim 20 \mathrm{~ns}$; though all the testing described in this paper was conducted with a $5 \mathrm{~ns}$ nearly flat in time (FIT) profile. Typical spatial and temporal profiles are shown in Figure $2 \mathrm{a}$ and $2 \mathrm{~b}$, respectively. The shot shown in Figure 2 was at an average fluence of $12 \mathrm{~J} / \mathrm{cm}^{2}$ with a beam contrast of $16 \%$ over the central $~ 85 \%$ of the beam.

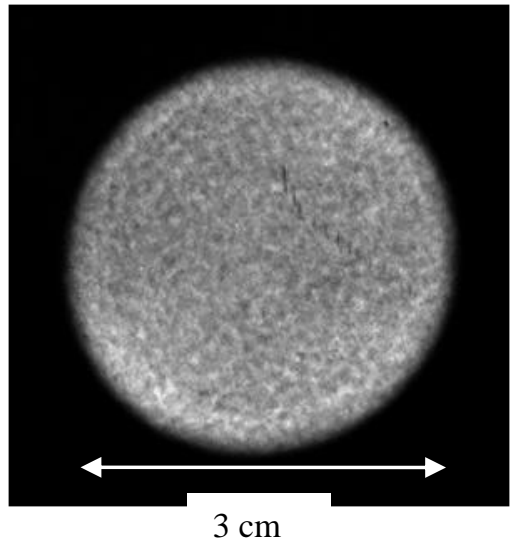

(a)

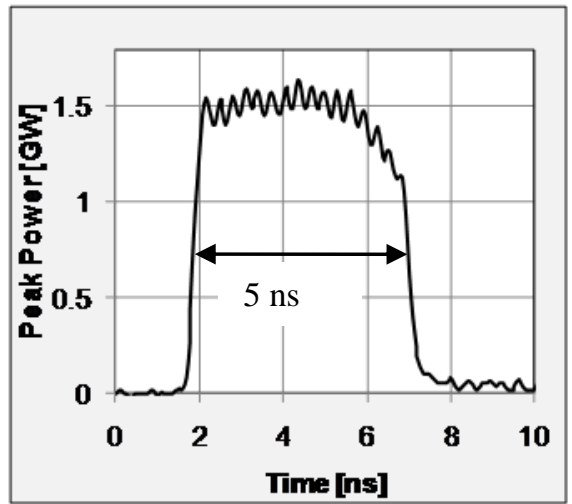

(b)

Figure 2. OSL spatial and temporal beam profiles.

The procedure followed for testing these samples requires multiple steps as shown in Figure 3. The sample is prepared with the scratches and then scanned using a commercial automated microscope, the Benchmark300 from View MicroMetrology, over an area that encompasses the OSL laser beam. The part is then installed into the sample chamber where it is kept at a pressure of $10^{-5}$ torr during the laser shot. A sample of the laser beam is measured with a calorimeter and also recorded with a scientific grade CCD to provide a spatial distribution of the fluence on the part. An additional beam sample is sent to a calibrated photodiode to record the temporal profile of the shot. The laser beam that is transmitted through the part is also recorded in a relay plane of the sample to provide the means to register the local fluence on the shot. The part is rescanned with the microscope. At this point the data is available for processing to obtain the both the number of initiations and the location of the damage and the size of the scratch where damage was initiated. These steps are repeated as needed for additional shot cycles.

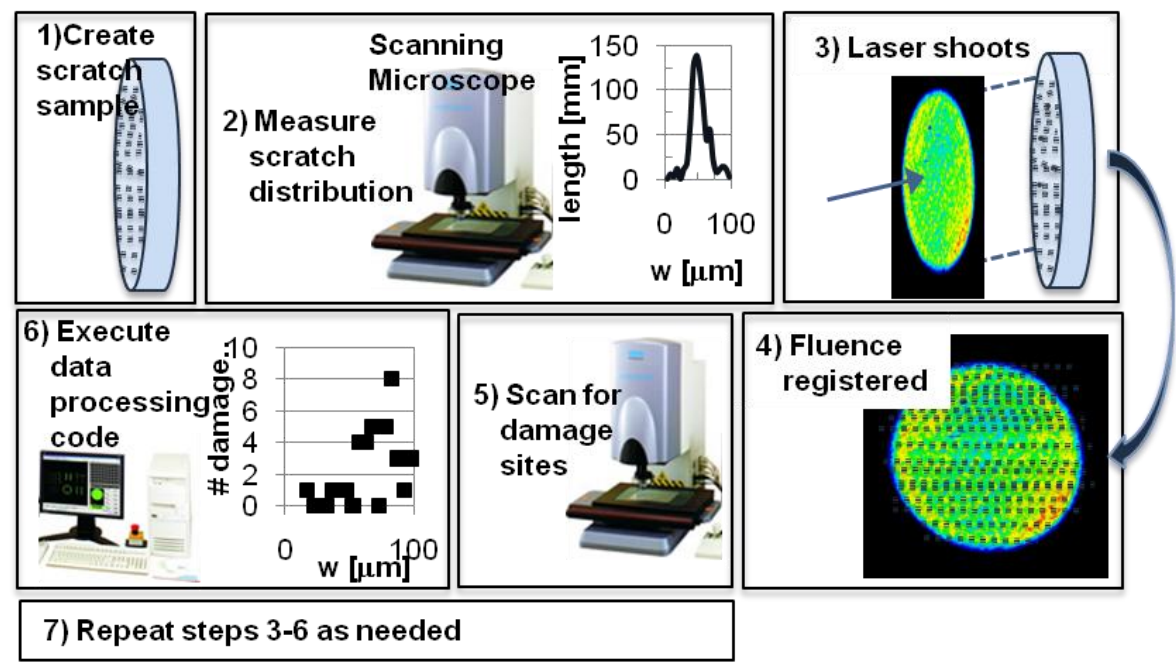

Figure 3. Procedure for damage testing. 


\section{DATA ANALYSIS METHODS}

\subsection{Data overview}

The acquisition of micrographs covering the $\sim 8 \mathrm{~cm}^{2}$ OSL beam area results in $\sim 1000$ individual images with a resolution of $\sim 2 \mu \mathrm{m}$. The challenge presented by this $165 \mathrm{Mb}$ image set is how to extract both scratch morphology and damage sites. This was first accomplished by assembling the images into a montage and manually measuring the scratch lengths and scratch widths. The next step was to compare the initial montage to the post shot montage and manually count the damage sites as a function of the scratch width. In fact the assembled montages can allow a fast comparison between different surface treatments; this is illustrated in figure 4 where figure 4 a shows the final results of a three shot series at $8 \mathrm{~J} / \mathrm{cm}^{2}, 10 \mathrm{~J} / \mathrm{cm}^{2}$, and $12 \mathrm{~J} / \mathrm{cm}^{2}$ on a sample prepared with 1997 -era scratch density, and figure $4 \mathrm{~b}$ shows the final results of a three shot series at $12 \mathrm{~J} / \mathrm{cm}^{2}$ on a similarly-prepared surface treated with AMP. Both surfaces have similar scratch distributions yet the AMP surface exhibits no damage, in spite of the more severe test sequence. In contrast, the unAMP'd surface has hundreds of initiated and growing damage sites, including many that initiated before the final shot.

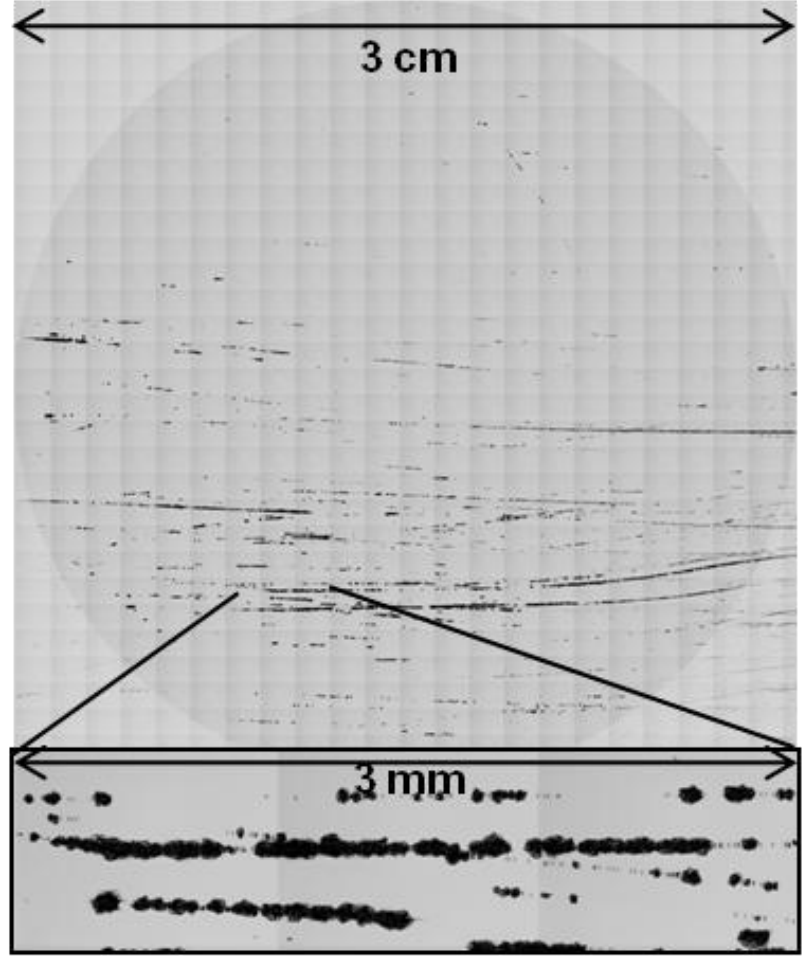

(a)

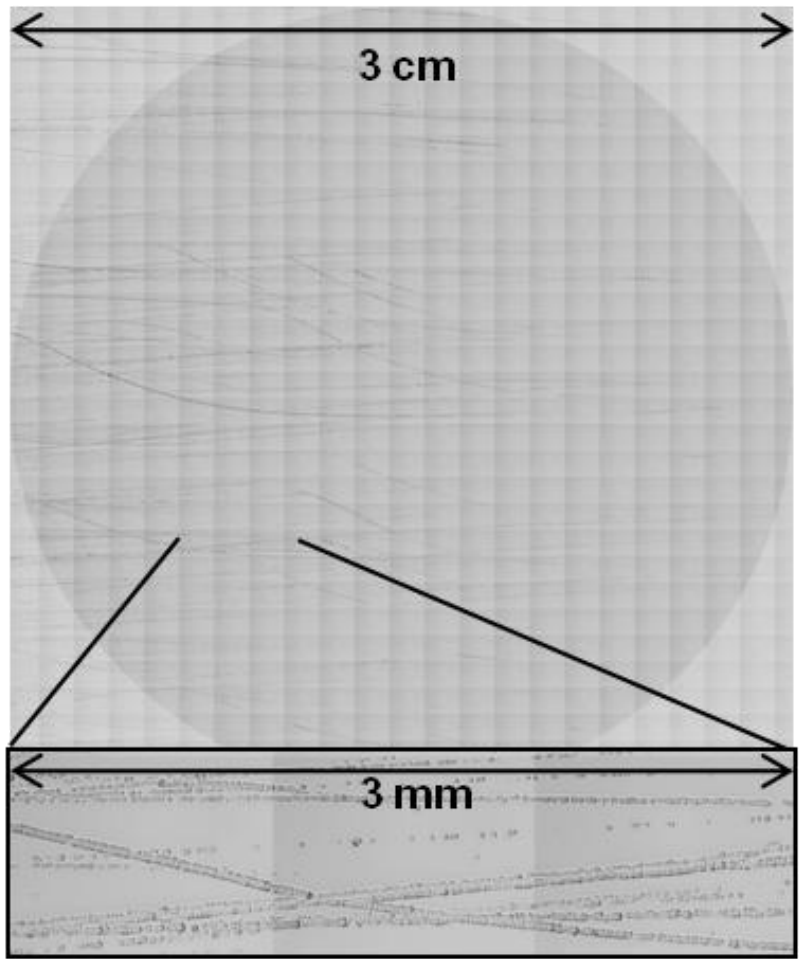

(b)

Figure 4. Comparison of montages of 2 surfaces after a 3 laser shot series.

\subsection{Scratch and damage analysis}

The manual procedure described in the previous section is tedious and time consuming. In an effort to develop programs to speed up and automate the analysis it was noted that detection of damage sites could be facilitated by allowing initiated sites to grow before trying to detect them. That this was a viable approach is illustrated in Figure 5 which compares the image of a small site after initiation at $13 \mathrm{~J} / \mathrm{cm}^{2}$ to the same location after two additional shots at a slightly lower fluence. A data processing code, Automated Scratch Initiation Recognition (ASIR), was written in ImageJ, an open source Java based image analysis tool from the U. S. National Institutes of Health, to automate scratch width and length measurement along with damage site detection. This code was benchmarked against the results of manual detection and was found to reproduce the results in $\sim 5$ minutes versus $\sim 3$ hours required for manual detection. The code first removes background lighting non-uniformities from the micrographs and then makes a pass through all the images using heavy thresholding to detect probable damage sites, letting the user decide whether it should be tabulated as 
damage. It makes an additional pass through the images using light thresholding to locate and catalog scratches, when an object is detected it is fit to an ellipse to measure length and width. The width distribution is then converted to a length in a width bin.

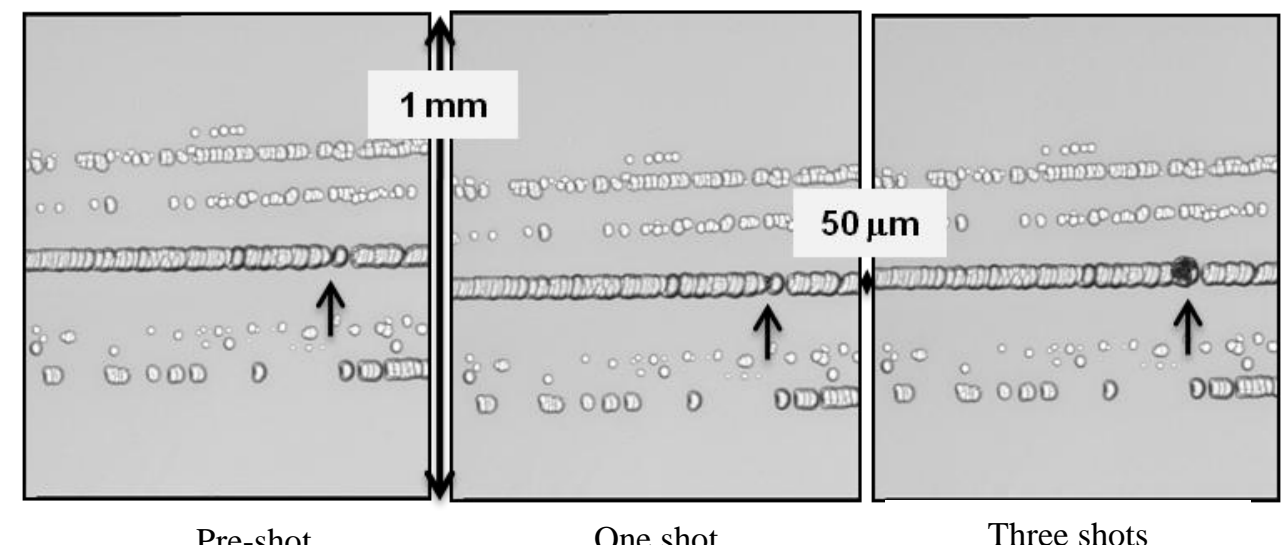

Pre-shot

One shot

Three shots

Figure 5. Micrographs showing initiation \& growth.

\section{RESULTS}

\subsection{Damage initiation on scratches}

After a three shot test series, histograms of the scratch length distribution and the damage count distribution versus scratch width are calculated to evaluate the performance of the test surface. A typical output from ASIR for a scratch sample with the 2010 AMP treatment is shown in Figure 6. The plot shows the total length of scratches measured in a $5 \mu \mathrm{m}$-size width bin (left-hand axis), and the number of initiations found in each width bin (right-hand axis). These histograms are typical for the 2010 AMP process in that most of the scratches are between $20 \mu \mathrm{m}$ and $70 \mu \mathrm{m}$ in width and that very little or frequently no damage is seen on scratches less than $60 \mu \mathrm{m}$ in width.

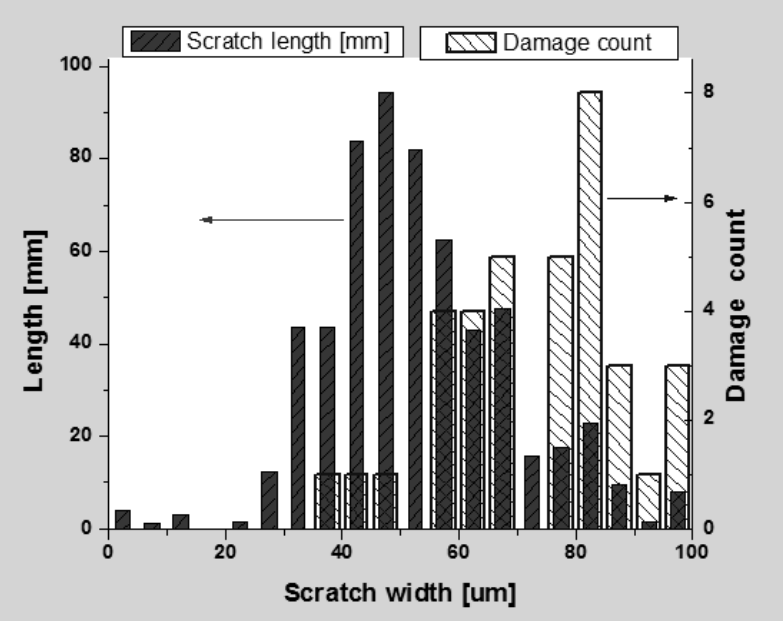

Figure 6. Histograms for scratch distribution and damage initiation count from ASIR.

Since the desired outcome of these experiments is to be able to predict the number of expected damage initiations on a real optic under expected use conditions it will require sufficient testing on these subscale parts to yield, preferably, a 
functional relationship of the number of initiations vs. scratch width. But this will yield only one piece of the needed knowledge since in addition it will be necessary to know how this data which we have obtained at $12 \mathrm{~J} / \mathrm{cm}^{2}$ scales vs. fluence. If both these relations are determined there is yet one more piece of information that must be obtained before it is possible to predict the number of initiations that would be expected on an optic: the scratch distribution of the optic.

Similarly prepared samples were tested in the same way and after tabulation of results from over $25 \mathrm{~m}$ of total scratch length the probable number of damage initiations per unit length versus the scratch width has been determined at $12 \mathrm{~J} / \mathrm{cm}^{2}$; this linear density [4] $\rho_{12}$ is plotted in Figure 7a. Similar experiments were undertaken on samples with

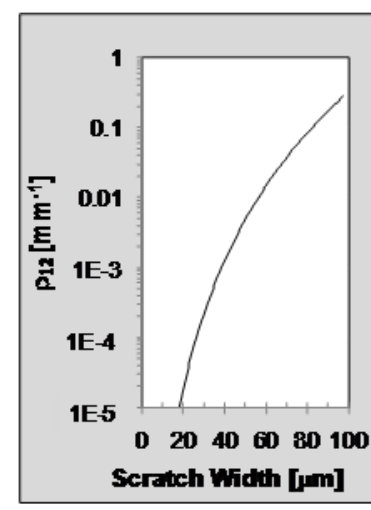

(a)

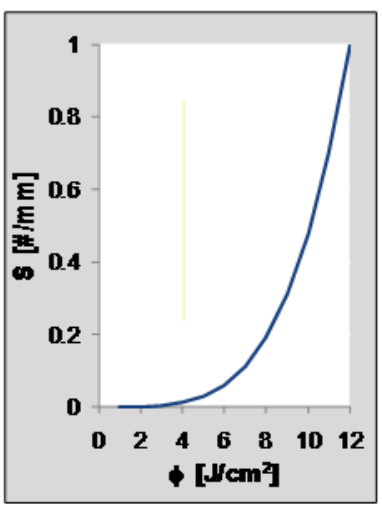

(b)

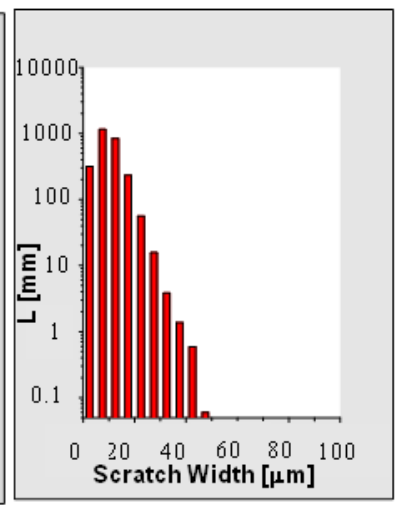

(c)

Figure 7. Relationships for initiation, fluence and scratch distributions.

surfaces having a preparation similar to the 2007 surfaces to obtain a fluence dependence for scratches of a fixed width, $\mathrm{S}$, which is shown in Figure 7b. The current assumption is that the same dependence will be found for the AMP treated scratches; this is an active area of investigation where the testing so far supports the assumption. The final plot shown in Figure 7c shows the scratch distribution measured on a single large scale optic; additional sampling of full scale optics is expected to yield similar data.

These three relationships would then be used to predict the number of initiations (n) to be expected for the optic in the use condition as follows:

$$
\mathrm{n}=S(\phi) \sum_{W} L(W) \rho_{12}(W)
$$

\subsection{Damage growth on scratches}

The growth rate of damage initiated on scratches was tested by exposing samples prepared as per Figure 1 to the OSL beam at $\sim 9 \mathrm{~J} / \mathrm{cm}^{2}, 5 \mathrm{~ns}$ FIT for a sequence of 10 shots. The immediate objective was to determine if these sites would grow in the manner previously measured for damage sites not located on scratches [6]. To evaluate the growth, we followed the diameter of a few sites that were somewhat isolated from other damage sites along a scratch; the diameter measured after each shot is plotted for two of these sites in figure 8a and 8b, along with the diameter of an isolated site grown under similar conditions [7] in Figure 8c. All three of these plots can be fitted to an exponential with growth coefficient, $\alpha$, near 0.2 , suggesting that damage that initiates on scratches located on the exit surface will grow at a rate similar to the isolated sites previously studied. There is some indication that as sites on scratches grow the aspect ratio may be influenced by the underlying scratch morphology. This behavior is shown in Figure 9 for two sites grown at local fluences of $\sim 8 \mathrm{~J} / \mathrm{cm}^{2}$. The sequence shown in Figure $9 \mathrm{a}$ is essentially indistinguishable from growth on isolated sites displaying aspect ratios near one, whereas the sequence shown in Figure $9 \mathrm{~b}$ is growing in an elongated fashion. 


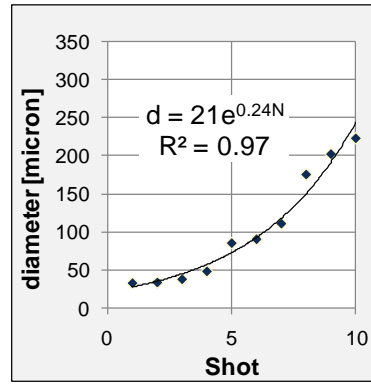

(a)

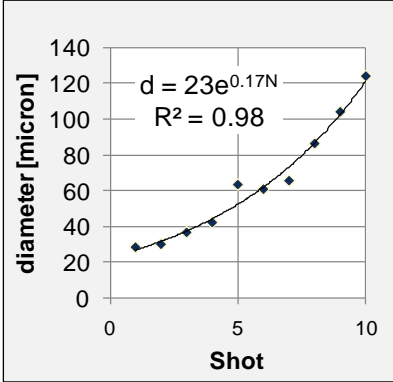

(b)

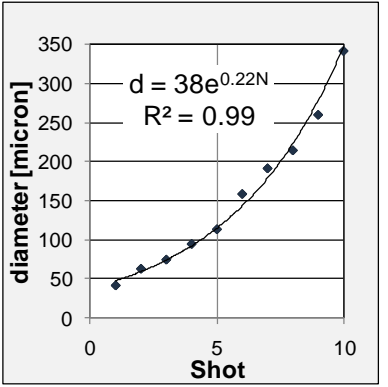

(c)

Figure 8. Damage growth on initiated scratches and on an isolated site.
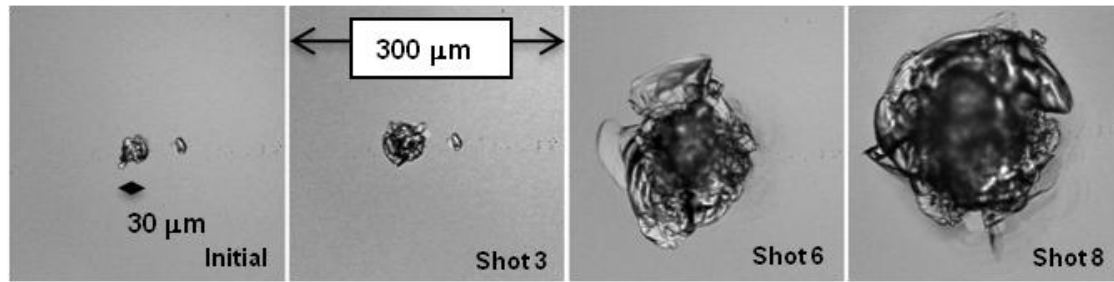

(a)
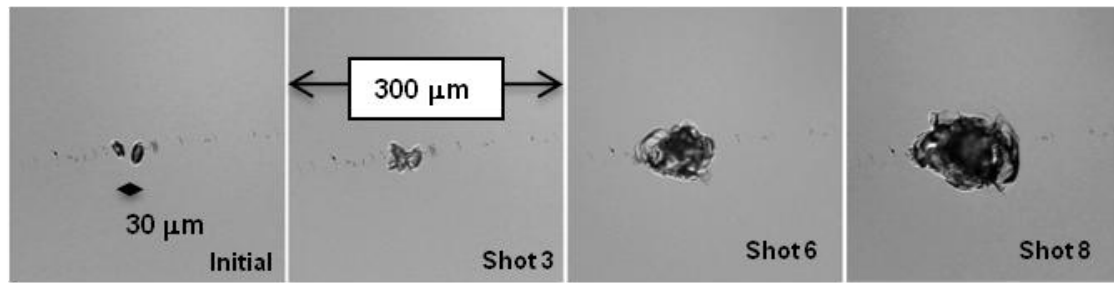

(b)

Figure 9. Micrographs of two sites grown at $8 \mathrm{~J} / \mathrm{cm}^{2}$.

\section{CONCLUSIONS}

We have developed a technique to measure the damage initiation density on scratches as a function of their width and length. To address the expected performance from the testing of subscale parts we have developed new methods to produce these subscale parts with representative scratches and with distributions that provide sufficient scratch lengths and widths that can be tested with a high quality $3-\mathrm{cm}$ diameter laser beam. To address the testing and subsequent analysis we have developed code for measuring damage initiations and scratch width and length distributions with a fast throughput. Both damage initiation linear densities on scratches of varying widths and fluence dependence of the initiations have been studied. Future work is planned to explore the linear initiation density dependence on the temporal pulse shape. [8], [9] Functional relationships that can be used to predict performance of full scale parts have been obtained. We have used this technique to evaluate a number of mitigation techniques and have shown that the 2010 AMP treatment has greatly reduced the number of damage initiations on the exit surface of fused silica optics used at $351 \mathrm{~nm}$. Work on exit surface growth of sites initiated on these subscale parts indicates that for $5 \mathrm{~ns}$ pulse widths the dependence of growth on fluence can be predicted from the rules governing growth of isolated sites. 


\section{ACKNOWLEDGEMENTS}

The authors would like to acknowledge the able contributions of the OSL staff (E. Donohue, W. Hollingsworth, R. Luthi, J. Prior, J. Vickers, T. Weiland) in conducting the laser tests. This work was performed under the auspices of the U. S. Department of Energy by Lawrence Livermore National Laboratory under contract DE-AC52-07NA27344.

\section{REFERENCES}

[1] Miller, P., Suratwala, T., Wong, L., Feit, M., Menapace, J., Davis, P., and Steele, R., "The distribution of subsurface damage in fused silica," Proc. SPIE 5991, (2005)

[2] Suratwala, T. I., Miller, P. E., Bude, J. D, Steele, Shen, N., Monticelli, M. V., Feit, M. D., Laurence, T.A., Norton, M. A., Carr, C. W., and Wong, L. L., "HF-based etching processes for improving laser damage resistance of fused silica optical surfaces," J. Am. Ceram. Soc. 93, doi: 10.1111/j.1551-2916,2010.014112.x (2010).

[3] Suratwala, T. I., Miller, P. E., Bude, J. D, Steele, Shen, N., Monticelli, M. V., Feit, M. D., Laurence, T.A., Norton, M. A., Carr, C. W., and Wong, L. L., "HF-based etching processes for improving laser damage resistance of fused silica optical surfaces," , these proceedings.

[4] Carr, C. W., Feit, M. D.,Nostrand, M. C. and J. J. Adams, "Techniques for qualitative and quantitative measurement of aspects of laser in-induced damage important for laser beam propagation," Meas. Sci. Technol. 17 (7), 1958-1962 (2006).

[5] Nostrand, M. C., T. L. Weiland, T. L., Luthi, R. L., Vickers, J. L., Sell, W. D., Stanley, J. A., Honig, J., Auerbach, J. Hackel, R. P.,and P. J. Wegner, "A large aperture, high energy laser system for optics and optical component testing," Proc, SPIE 5273, 325, (2003)

[6] Norton, M. A., Donohue, E. E., Feit, M. D., Hackel, R.P., Hollingsworth, W.G., Rubenchick, A.M., and M. L. Spaeth, "Growth of laser damage in $\mathrm{SiO}_{2}$ under multiple wavelength irradiation," Proc. SPIE 5991, (2005)

[7] Negres, R. A., Norton, M. A., Liao, Z. M., Cross, D. A., Bude, J. D., and Carr, C. W. "The effect of pulse duration on the growth of laser-induced damage sites at $351 \mathrm{~nm}$ on fused silica surfaces." Proc. SPIE 7504, 2009.

[8] Carr, C. W., Trenholme, J. B., and M. L. Spaeth, "Effect of temporal pulse shape on optical damage," Appl. Phys. Lett. 90 (4), (2007).

[9] Carr, C. W., Matthews, M. J., Bude, J. D., and M. L. Spaeth, "The effect of laser pulse duration on laser-induced damage in $\mathrm{KDP}$ and $\mathrm{SiO}_{2}$, , Proc. SPIE 6403 , (2006) 